\title{
Lung structure and function of infants with recurrent wheeze when asymptomatic
}

\author{
C.J. Llapur*,\#, T.M. Martínez*, C. Coates*, C. Tiller*, J.L. Wiebke*, X. Li" ${ }^{\star}$, \\ K. Applegate ${ }^{+}$, H.O. Coxson ${ }^{\S, f}$ and R.S. Tepper*
}

ABSTRACT: Infants with recurrent wheeze have repeated episodes of airways obstruction; however, relatively little is known about the structure and function of their lungs when not symptomatic. The current authors evaluated whether infants with recurrent wheeze have smaller airway lumens or thickened airway walls, as well as decreased airway function.

High-resolution computed tomography images $1 \mathrm{~mm}$ thick were obtained at three anatomic locations at an elevated lung volume and at functional residual capacity. Forced expiratory flows were also measured in subjects with recurrent wheeze.

Airway lumen, wall areas and lung tissue density were not significantly different for recurrent wheeze $(n=17)$ and control $(n=14)$ subjects; however, subjects with recurrent wheeze had lower forced expiratory flows than predicted. Similar findings were obtained when subjects were grouped by exposure to tobacco smoke.

These findings indicate that infants with recurrent wheeze, as well as exposure to tobacco smoke, have lower airway function when not symptomatic. The lower forced expiratory flows may result from a degree of airway narrowing that could not be resolved with the methodology employed or from other mechanisms, such as more collapsible airways or decreased pulmonary elastic recoil.

KEYWORDS: Airway structure, infants, lung function, lung tissue density

$\mathbf{R}$ ecurrent wheezing is a common respiratory problem early in life. Longitudinal studies have categorised recurrent wheezers into transient and persistent. Prior to the onset of recurrent wheezing, transient wheezers have lower lung function than neverwheezers and are more likely to have a respiratory history positive for exposure to tobacco smoking (ETS) [1] than positive for family asthma/allergy. Persistent wheezers have premorbid lung function similar to never-wheezers and are more likely to have history positive for family asthma/allergy and ETS. These findings, as well as the findings that ETS is related to lower airway function, have suggested that different mechanisms contribute to the airway pathophysiology among infants and toddlers with recurrent wheezing $[2,3]$.

Infants and toddlers with recurrent wheeze (RW) have repeated episodes of airways obstruction severe enough to produce wheezing; however, relatively little is known about the structure and function of their lungs between episodes of wheezing, when they are not symptomatic. High-resolution computed tomography (HRCT) imaging of adults and older children with various lung diseases has been an important modality for assessing lung structure in vivo [4-9]. It has recently been reported that infants and toddlers with cystic fibrosis (CF) who are not acutely symptomatic have thickened airway walls and narrowed airway lumens when assessed by HRCT imaging. In addition, the quantitative measures of airway structure correlated with airway function [10].

In the current study, it was hypothesised that subjects with RW would have decreased airway function, as well as smaller airway lumens and/ or thickened airway walls, when they were without acute respiratory symptoms. In addition, the present authors hypothesised that structural differences may relate to history of ETS.

\section{METHODS}

\section{Subjects}

In total, 17 infants and toddlers aged between 4 months and 2.3 yrs with a respiratory history of at least three episodes of wheeze were evaluated when they were clinically stable outpatients without respiratory symptoms for $\geqslant 3$ weeks. Subjects with RW were recruited from the pulmonary clinic at the James Whitcomb Riley
AFFILIATIONS

Depts of *Paediatric Pulmonology

and Critical Care,

"Biostatistics and

${ }^{+}$Radiology, Indiana University

Medical Center, Indianapolis, IN, USA.

\#Dept of Paediatric Pulmonology, Hospital del Niño Jesús, Faculty of Medicine, National University of Tucumán, Tucumán, Argentina. ${ }^{\S}$ Dept of Radiology, Vancouver General Hospital, and

${ }^{f}$ James Hogg iCAPTURE Centre for Cardiovascular and Pulmonary Research, St Paul's Hospital, University of British Columbia, Vancouver, BC, Canada.

\section{CORRESPONDENCE}

R.S. Tepper, Dept of Paediatrics, Section of Paediatric Pulmonology, James Whitcomb Riley Hospital for Children, 702 Barnhill Drive, Room 4270, Indianapolis, IN 46202, USA. Fax: 13172745791

E-mail: rtepper@iupui.edu

Received:

August 142007

Accepted after revision:

August 042008

\section{SUPPORT STATEMENT}

This study was supported by National Institutes of Health (Bethesda, MD, USA) research grant 54062.

STATEMENT OF INTEREST A statement of interest for H.O.

Coxson can be found at www.erj.ersjournals.com/misc/ statements.shtml 
Hospital for Children (Indianapolis, IN, USA) by one of the paediatric pulmonologists or the pulmonary research nurse, after reviewing the clinic chart to determine that the infant was full-term at birth, had no congenital cardiorespiratory anomalies and was not being treated for gastro-oesophageal reflux, aspiration or CF. Approximately 60 patients were approached in order to obtain the group of subjects that participated in the study. Infant pulmonary function testing was performed the first day; a HRCT was performed 48-72 h later. Out of the 17 subjects, 12 were on inhaled corticosteroids, 9 used $\beta$-agonists on a nonscheduled basis and 11 had been hospitalised for respiratory problems at least once prior to the present study. Additionally, 14 control infants and toddlers were recruited in the radiology department of the same hospital, from patients undergoing computed tomography (CT) for nonrespiratory medical problems; results from these subjects were previously presented [10]. The subject's family history was positive for asthma/allergy if either of the parents or any siblings were affected. ETS was assessed by questionnaire, as well as nicotine and cotinine levels measured from the infant's hair [11]. The Institutional Review Board of the James Whitcomb Riley Hospital for Children approved the study and informed consent was obtained from parents.

\section{HRCT}

Sedated subjects (50-75 mg. $\mathrm{kg}^{-1}$ chloral hydrate) were evaluated in the supine position [10]. During induced respiratory pauses at a lung volume defined by an airway pressure of $20 \mathrm{cmH}_{2} \mathrm{O}(V 20)$ and at passive functional residual capacity (FRC), 1-mm thick images $(120 \mathrm{kVp}, 40 \mathrm{mAs})$ were acquired at three anatomic locations: the top of the aortic arch, the level of the carina and $\sim 1 \mathrm{~cm}$ above the diaphragm. Images were reconstructed using a high spatial frequency reconstruction algorithm, a 1,024 ×1,024 matrix and the smallest field of view $(15-25 \mathrm{~cm})$. Total radiation exposure was estimated to be $0.035 \mathrm{mSv}$ for the six images. Images were analysed by two different operators blinded to subject grouping; the analysis of the images was not randomised. From images obtained at V20, all airways with long/short internal diameter ratio $<2.2$ were analysed. Airway dimensions were measured using custom software (EmphylxJ; Thoracic Imaging Group, Vancouver Hospital, Vancouver, BC, Canada) [12]. The total airway area, lumen area (LA) and the area of adjacent artery (AA) were measured, and the airway wall area (WA) was calculated [10, 13]. From the images obtained at FRC, lung parenchyma was automatically segmented from the chest wall and large central blood vessels using a modified bordertracing algorithm with a prior position-knowledge algorithm using CT values between -1,000 (air) and -200 Hounsfield Units (HU). Lung density $\left(\mathrm{g} \cdot \mathrm{mL}^{-1}\right)$ was calculated by adding 1,000 to the HU of each voxel to convert to CT value $[10,14]$.

\section{Airway function}

Airway function was measured in sedated $\left(50-75 \mathrm{mg} \cdot \mathrm{kg}^{-1}\right.$ chloral hydrate) RW subjects using the raised volume rapid thoracic compression technique [15]. Following baseline measurements, infants inhaled nebulised albuterol $(2.5 \mathrm{mg}$ in $2 \mathrm{~mL}$ normal saline) and measurements were repeated $10 \mathrm{~min}$ after the aerosol. Forced vital capacity (FVC) and forced expiratory flows at 50\% and $75 \%$ expired volume (FEF50 and FEF75, respectively) were measured, as well as the forced expiratory flow between $25 \%$ and $75 \%$ expired volume (FEF25-75) and the forced expiratory volume in the initial $0.5 \mathrm{~s}$ (FEV0.5). Values from the best pre- and postbronchodilator curves were expressed as z-scores using a reference population from the authors' laboratory at the James Whitcomb Riley Hospital for Children [15].

\section{Statistical analysis}

Demographics for RW and control groups were compared using one-way ANOVA and categorical variables were compared with Fisher's exact test. For the analyses of differences in the airways between groups, repeated-measures ANOVA by the methodology of mixed models was used, to account for the correlation of multiple measurements within subjects. Potential confounding effects of age, sex and somatic size were included in the models, but were not found to be significant. Mean values for tissue density were compared using one-way ANOVA. A two-sided p-value $<0.05$ was considered statistically significant for all tests.

\section{RESULTS \\ Demographics}

Group data for RW and control subjects are summarised in table 1. Questionnaire data were obtained from all $17 \mathrm{RW}$ infants and 11 out of 14 controls. The groups were not significantly different in terms of age, sex, weight or length, and there were no significant differences in history of family asthma/allergy or ETS.

\section{Airway measurements}

The total airway size (LA plus WA) for each of the airways measured is illustrated in figure 1 . No significant differences

TABLE 1 Patient demographics: control versus recurrent wheeze (RW)

\begin{tabular}{lccc} 
& Control & RW & p-value \\
\hline Subjects $\mathbf{n}$ & 14 & 17 & 0.97 \\
Age months & $14.5(6-29)$ & $14.6(4-28)$ & 0.44 \\
Sex (M/F) $\mathbf{n}$ & $8 / 6$ & $10.5(8.3-13.7)$ & 0.1 \\
Weight $\mathbf{k g}$ & $9.5(6.8-12)$ & $76(63.3-91)$ & 0.94 \\
Body length $\mathbf{~ m}$ & $75.7(67.5-82.7)$ & $8 / 9$ & 0.18 \\
Tobacco smoking exposure (yes/no) $\mathbf{n}$ & $8 / 3$ & $7 / 10$ & 0.7 \\
Family history of asthma/allergy (yes/no) $\mathbf{n}$ & $2 / 9$ & & \\
\hline
\end{tabular}

Data are presented as mean (range), unless otherwise stated. M: male; F: female 


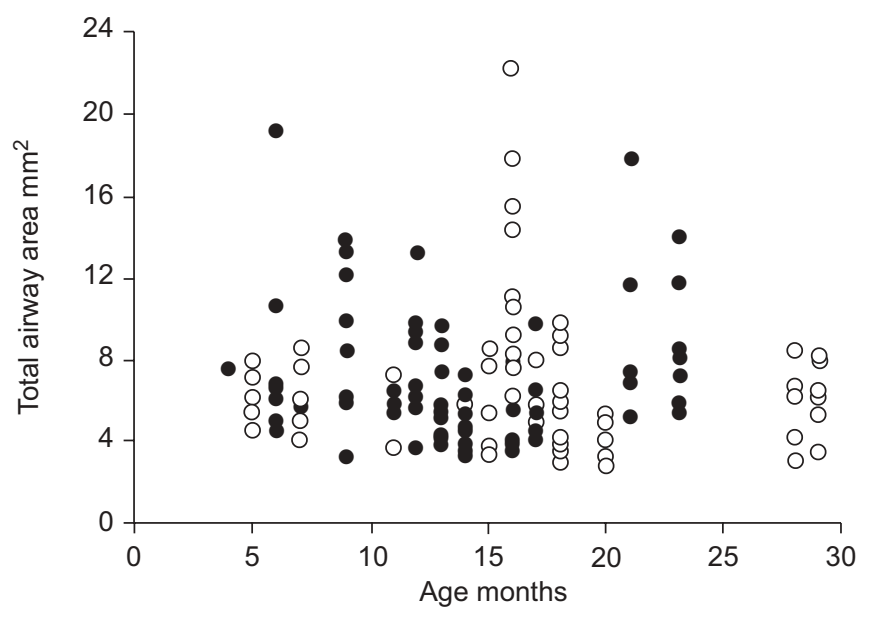

FIGURE 1. Total airway area versus age for all of the individual airways measured from high-resolution computed tomography images obtained at an airway pressure of $20 \mathrm{cmH}_{2} \mathrm{O}$ for control subjects $(O)$ and subjects with recurrent wheeze $(\bullet)$. There was no significant difference in the number of airways measured $(p=0.15)$ or the total airway area for the two groups $(p=0.76)$

were found between the two operators who measured LA $(p=0.93)$ and WA $(p=0.92)$; the interobserver correlation coefficient was 0.95 . There was no significant difference in the number of airways measured for RW and control subjects (mean (range) 7 (3-20) versus $9.3(4-19) ; p=0.15)$. There was also no significant difference in total airway size for the two groups $(\mathrm{p}=0.76)$.

For each airway, the ratios of LA to AA and of WA to AA were calculated and $\log$ transformed for analysis, as previously described by DE JONG et al. [13]. Figure 2a illustrates individual values of $\log (\mathrm{LA} / \mathrm{AA})$ for RW and control subjects. For both groups, $\log (\mathrm{LA} / \mathrm{AA})$ did not vary with age $(\mathrm{p}=0.64)$. There was also no significant difference in $\log$ (LA/AA) for RW and control subjects $(p=0.53)$. Figure $2 b$ illustrates individual values of $\log$ (WA/AA) for RW and control subjects. For both groups, $\log (\mathrm{WA} / \mathrm{AA})$ did not vary with age $(\mathrm{p}=0.29)$, and there was no significant difference in $\log (\mathrm{WA} / \mathrm{AA})$ for RW and control subjects $(p=0.38)$.

The effect of ETS upon airway measurements from HRCT images was evaluated with all subjects combined (RW and control). Smoking exposure data obtained by questionnaire was available for all RW subjects and for 11 out of 14 control subjects, while hair samples for nicotine and cotinine were available from 27 out of 31 subjects. Subjects with positive ETS by history had significantly higher levels of hair nicotine (17.2 versus $0.39 \mathrm{ng} \cdot \mathrm{mg}^{-1}$ hair; $\left.\mathrm{p}<0.02\right)$ and cotinine $(0.97$ versus $0.07 \mathrm{ng} \cdot \mathrm{mg}^{-1}$ hair; $\left.\mathrm{p}<0.001\right)$. When using hair cotinine data, there were no significant differences between exposed and nonexposed subjects for $\log (\mathrm{LA} / \mathrm{AA})(\mathrm{p}=0.66)$ or $\log$ (WA/ AA) $(p=0.88$; fig. 3). Similar results were obtained using hair nicotine or questionnaire data.

\section{Lung density}

A cumulative distribution of tissue densities measured from the HRCT images obtained at $V 20$ are illustrated in figure 4 for the two groups. Using the mean tissue density, the analysis
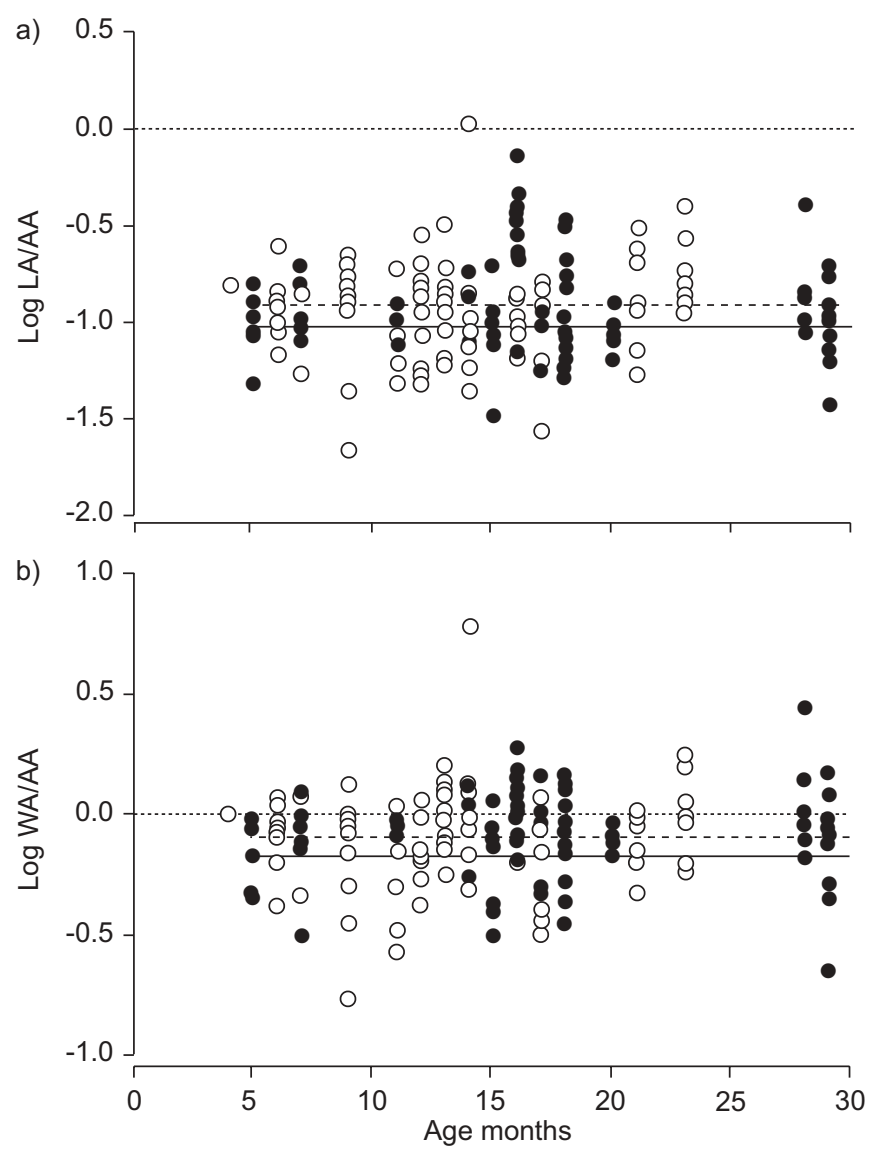

FIGURE 2. a) Airway lumen area (LA) and b) airway wall area (WA) assessed using high-resolution computed tomography at a lung volume defined by an airway pressure of $20 \mathrm{cmH}_{2} \mathrm{O}$. No significant differences were found between the subjects with recurrent wheeze (RW; - ) and control subjects $(O)$ when the ratios of $L A$ to arterial area (AA) and of WA to AA were adjusted for age and sex. - - - -: average for controls; —-: average for RW subjects.

assessed differences and interactions between groups, including the level at which the three HRCT slices were obtained in the same subject. There were no significant differences between RW and control subjects (table 2). In addition, there were no significant differences found between RW and control subjects when images were evaluated at FRC.

When subjects were grouped by ETS, there was no significant difference in the lung density between the two groups when exposure was assessed by hair cotinine level (table 2), as well as by nicotine level or questionnaire data.

\section{Pulmonary function}

Measurements were obtained in the $17 \mathrm{RW}$ subjects. The group mean z-score for FEF75 was -0.77 , which was significantly lower than zero $(p<0.003)$. Following the inhaled bronchodilator, there was no significant change from baseline in the group's mean z-scores for any of the pulmonary function parameters: FEF75 -0.77 versus $-0.70(\mathrm{p}=0.83)$; FEV0.5 -0.89 versus $-1.2(\mathrm{p}=0.57)$; FEF50 -0.89 versus $-0.97(\mathrm{p}=0.84)$; FEF25-75 -1.05 versus $-0.11(\mathrm{p}=0.88)$; and FVC -0.8 versus $-1.0(\mathrm{p}=0.70)$. Among RW subjects, airway size from HRCT images did not correlate with airway function. 

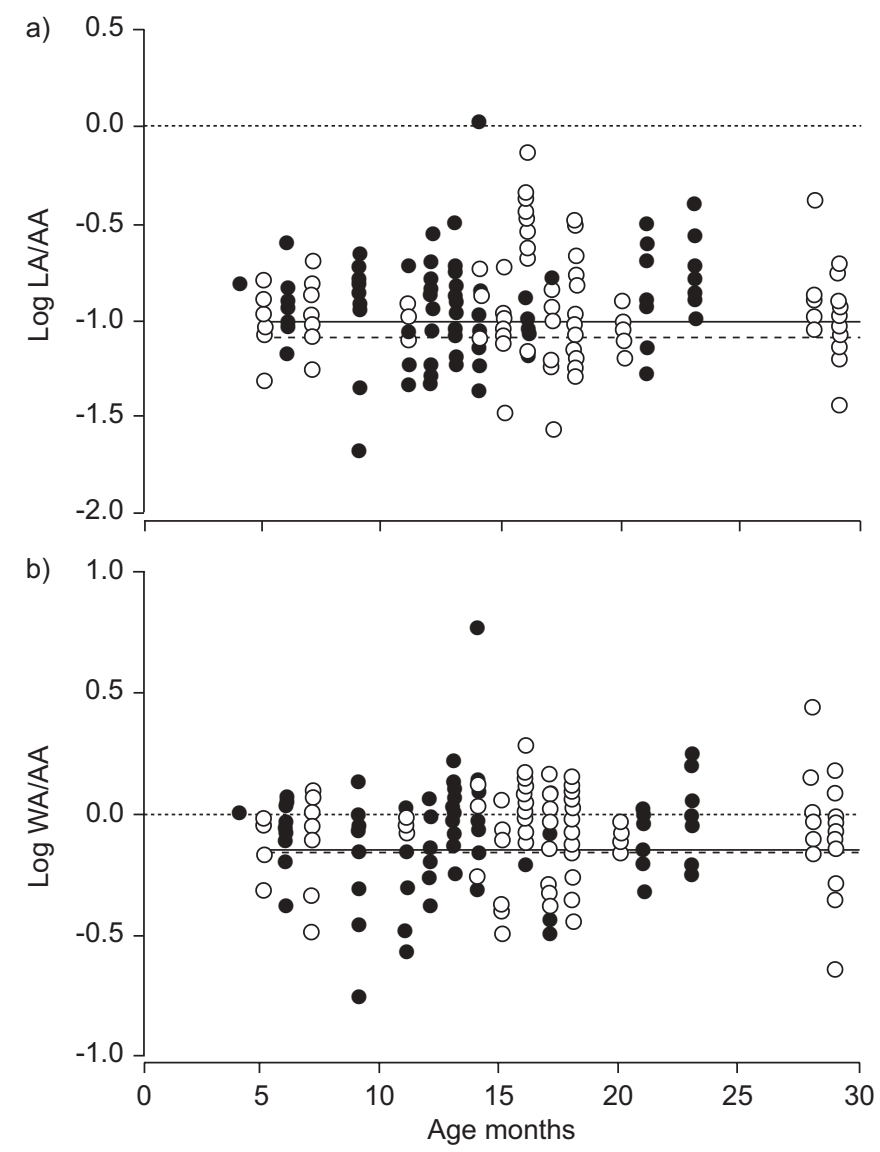

FIGURE 3. a) Airway lumen area (LA) and b) airway wall area (WA) assessed using high-resolution computed tomography at a lung volume defined by an airway pressure of $20 \mathrm{cmH}_{2} \mathrm{O}$ in subjects with exposure to tobacco smoking (ETS; - ) versus infants with no ETS $(\bigcirc)$, as assessed by hair cotinine levels. No significant differences were found between the groups when the ratios of LA to arterial area (AA) and of WA to AA were adjusted for age and sex. - . - - average for subjects with no ETS; —: average for subjects with ETS.

\section{DISCUSSION}

The current authors evaluated whether infants with a history of RW had smaller airway calibre, thickened airway walls and decreased airway function when they were without respiratory symptoms. It was found that airway calibre, assessed by a limited number of HRCT lung slices obtained at elevated lung volume, did not differ between RW and control subjects. The infants with RW appeared to have airways of normal calibre when assessed by HRCT when they were asymptomatic; however, they did have lower forced expiratory flows, which did not increase following an inhaled bronchodilator. Similar findings were obtained when subjects were divided by ETS rather than RW. The present findings indicate that infants with RW or ETS have decreased airway function when without respiratory symptoms. The lower forced expiratory flows may result from a degree of airway wall thickening that was not able to be resolved with the methodology employed or from mechanisms other than thickened airway walls, such as more collapsible airways or decreased pulmonary elastic recoil.

A limited number of HRCT slices were used, which assumes that comparable airways were sampled from all subjects. No

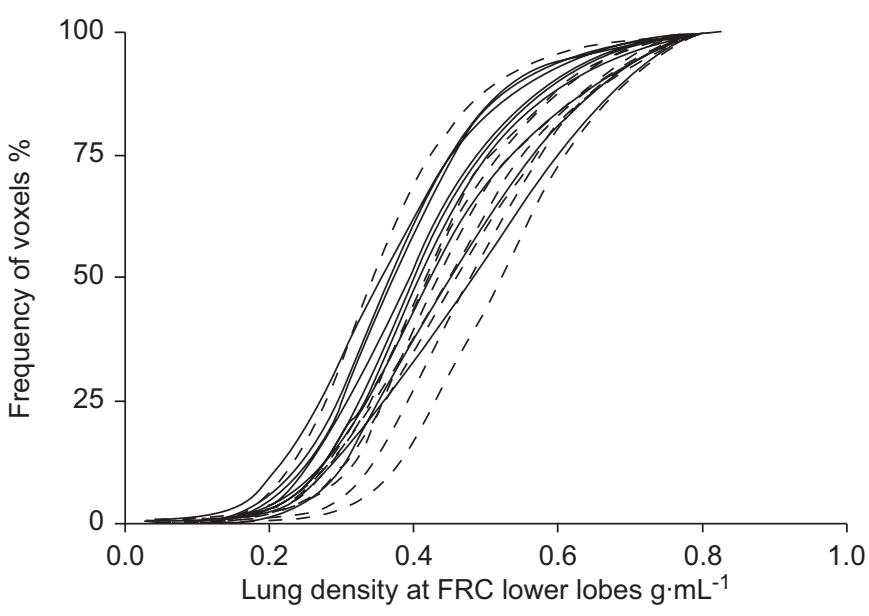

FIGURE 4. Cumulative distribution of the lung tissue densities from the highresolution computed tomography images obtained from the lower lobes during respiratory pause at functional residual capacity (FRC). No statistically significant differences were found between the subjects with recurrent wheeze (- - - ) and the control subjects $(-; p=0.4)$.

significant difference was found in the number of airways sampled for the RW and control subjects. In addition, there was no significant difference between the two groups in the overall size of the airways measured at an elevated lung volume, V20. These findings suggest that a comparable distribution of airways were examined for all subjects; however, an important limitation of the present study was the use of only a few HRCT slices for obtaining quantitative measurements. A previous study of older children with $\mathrm{CF}$ reported that decreasing the number of HRCT slices from a volumetric scan to $<10$ slices decreased the ability to detect an abnormal scan when assessed by an overall radiographic score [16]. A similar study using quantitative measures of airway size has not been performed and a different study in $\mathrm{CF}$ children did not find a correlation between CT scores and quantitative airway measurements [17]. Use of more lung slices, but with the same or lower radiation dose, would increase the number of airways evaluated; however, the lower radiation dose for each slice would probably decrease the resolution of the airway measurement.

It was previously found that asymptomatic infants with $\mathrm{CF}$ have thickened airway walls, smaller airway lumens and lower lung density at FRC [10] when assessed by the same methodology as used in the current study. The CF infants had more severe airways obstruction by pulmonary function testing than the current RW infants, as well as evidence for air trapping on the limited HRCT images, which was not present in the RW infants. Therefore, the negative HRCT findings in the RW infants with less severe airways obstruction may reflect less thickened airway walls in the RW infants, which could not be detected owing to limited power from the number of subjects evaluated and number of airways evaluated from each subject with only a few lung slices.

From the current mixed model results, the difference in log (LA/AA) for RW versus control was 0.0061 and the difference in $\log$ (WA/AA) was 0.010 after adjusting for age. There were 
TABLE 2 Lung tissue density: effects of recurrent wheeze (RW) and exposure to tobacco smoking (ETS)

\begin{tabular}{llllll} 
& RW & Control & p-value & ETS cot+ & ETS cot- \\
\hline Inspiratory & $0.26 \pm 0.01$ & $0.24 \pm 0.01$ & 0.12 & $0.26 \pm 0.01$ & $0.26 \pm 0.01$ \\
Expiratory & $0.45 \pm 0.01$ & $0.43 \pm 0.01$ & 0.22 & $0.43 \pm 0.02$ & $0.44 \pm 0.02$ \\
\hline &
\end{tabular}

about $3 \%$ and $5 \%$ power, respectively, to detect the small differences observed in the current study at a two-sided significance level of 0.05 . The study by DE JONG et al. [13] reported that airway $\mathrm{LA} / \mathrm{AA}$ and WA/AA were 1.92 and 1.45 times higher, respectively, in children with CF compared with controls. The current authors did not expect to see differences as large in the current study of infants with RW, and the differences observed corresponded to LA/AA and WA/AA values of 1.01 and 1.02 times higher in infants with RW than controls, which was not significant. However, to detect half of the differences in $\log$ (LA/AA) and $\log$ (WA/AA) observed between the $\mathrm{CF}$ group and the control group reported by DE JONG et al. [13] (0.14 and 0.081, respectively), the present study would have had $62 \%$ and $59 \%$ power. The log differences 0.14 and 0.081 correspond to LA/AA and WA/ AA being 1.38 and 1.21 times higher in the RW group compared with the control group. To detect two-thirds of the differences reported in the study by DE JONG et al. [13], the present study would have had $86 \%$ and $83 \%$ power for LA/ AA and WA/AA, respectively, and this would correspond to LA/AA and WA/AA being 1.55 and 1.28 times higher in the RW group compared with the control group.

The current authors are not aware of pathological data on airway size or wall thickness for infants with RW, particularly where the specimens were obtained when the subjects were asymptomatic. Bronchial biopsies from infants and toddlers with RW suggest that the reticular basement membrane (RBM) is not thickened at this very young age, although thickening may be present by several years of age $[18,19]$. In adults, RBM thickness has been found to correlate with airway wall thickness assessed by HRCT imaging; however, no correlation was found in children [9]. The limited autopsy data from infants dying from sudden infant death syndrome suggests that high exposure to maternal tobacco smoking results in thicker airway walls internal to the airway smooth muscle, but not an overall thickened airway wall area [20]. In addition, this finding was not present for all airways but limited to the largest airways evaluated: those airways with estimated luminal diameters between 0.6 and $1.2 \mathrm{~mm}$, or estimated luminal areas between 0.28 and $1.1 \mathrm{~mm}^{2}$. Similar to the light microscopic results, the present HRCT measurements included similarly sized airways and also did not show any difference in total wall area. Unlike light microscopic measurements of fixed tissues, HRCT in vivo measurements are not able to subdivide the components of the overall wall thickness and evaluate the area internal to the airway smooth muscle. However, the evaluation of airways from autopsied lungs may have been confounded by post mortem bronchoconstriction and airway oedema, as well as the lung volume at which the airways were fixed.

The RW group, as well as the group of infants with ETS, had lower forced expiratory flows than the predicted values based upon healthy controls previously evaluated [15]. These findings are consistent with previously reported studies using forced expiratory flows and/or resistance for infants with RW, as well as ETS [2, 15, 21, 22]. Besides increased wall thickness that narrows the airway lumen, potential mechanisms for the decreased airway function include increased airway tone, more collapsible airways and lower pulmonary elastic recoil pressure.

The absence of a significant improvement in forced expiratory flows following an inhaled bronchodilator suggests that the lower flows were not secondary to increased bronchial tone. Post-bronchodilator airway function was assessed $10 \mathrm{~min}$ after the inhaled bronchodilator, which could potentially have underestimated the bronchodilator response. However, the current authors' experience with methacholine (MCh) challenges in this subject age group has demonstrated that the same delivery of a bronchodilator completely reverses acute $\mathrm{MCh}$-induced bronchoconstriction within $10 \mathrm{~min}$. Therefore, it is not likely that a significant bronchodilator response in these infants was missed.

It was not possible to assess the distensibility of the airway wall from the HRCT images obtained at the two different lung volumes, as the slices at each lung volume did not include the same anatomic airways. Limited physiological data from infants with RW suggest that these infants may differ in the mechanical properties of the airway wall [23]; however, direct measurements of airway mechanics have not been obtained in these subjects. Lower pulmonary elastic recoil in infants with RW or ETS could also contribute to lower forced expiratory flows. In the current study, tissue density was measured at an elevated lung volume. Prenatal exposure of monkeys to nicotine results in an increased alveolar mean linear intercept and increased volume density of alveolar air assessed by light microscopy [24], which could potentially be evident by measurement of parenchymal density on HRCT scans, as observed in adults with emphysema [7, 25]. No differences in tissue density were found at an elevated lung volume (V20) for the RW or ETS infants compared with healthy controls and infants with no ETS; this finding is consistent with no difference in parenchymal tissue properties for the RW and ETS groups of infants evaluated. It was previously reported that ETS was not associated with an alteration of the passive elastic properties of the respiratory system [26]; however, the 
current authors are not aware of any published studies that directly assessed pulmonary elastic recoil in these subjects.

In conclusion, using high-resolution computed tomography images, no evidence was found for smaller airways, thickened airway walls or air trapping in infants with a history of recurrent wheeze when they were without respiratory symptoms. However, these subjects did have decreased forced expiratory flow that did not increase following an inhaled bronchodilator. Similarly, infants with a history of exposure to tobacco smoking showed no evidence for smaller airways or lower tissue density at functional residual capacity, while this group of subjects also had decreased forced expiratory flows. The lower forced expiratory flows may result from a degree of airway narrowing that could not be resolved with the methodology employed or from other mechanisms, such as more collapsible airways or decreased pulmonary elastic recoil.

\section{REFERENCES}

1 Martinez FD, Wright AL, Taussig LM, Holberg CJ, Halonen M, Morgan WJ. Asthma and wheezing in the first six years of life. The Group Health Medical Associates. N Engl J Med 1995; 332: 133-138.

2 Stocks J, Dezateux C. The effect of parental smoking on lung function and development during infancy. Respirology 2003; 8: 266-285.

3 Tepper RS, Williams-Nkomo T, Martinez T, Kisling J, Coates C, Daggy J. Parental smoking and airway reactivity in healthy infants. Am J Respir Crit Care Med 2005; 171: 78-82.

4 King GG, Müller NL, Paré PD. Evaluation of airways in obstructive pulmonary disease using high-resolution computed tomography. Am J Respir Crit Care Med 1999; 159: 992-1004.

5 Niimi A, Matsumoto H, Amitani R, et al. Airway wall thickness in asthma assessed by computed tomography. Relation to clinical indices. Am J Respir Crit Care Med 2000; 162: 1518-1523.

6 de Jong PA, Long FR, Wong JC, et al. Computed tomographic estimation of lung dimensions throughout the growth period. Eur Respir J 2006; 27: 261-267.

7 Coxson $\mathrm{HO}$, Rogers RM, Whittall KP, et al. A quantification of the lung surface area in emphysema using computed tomography. Am J Respir Crit Care Med 1999; 159: 851-856.

8 Jain N, Covar RA, Gleason MC, Newell JD Jr, Gelfand EW, Spahn JD. Quantitative computed tomography detects peripheral airway disease in asthmatic children. Pediatr Pulmonol 2005; 40: 211-218.

9 Saglani S, Papaioannou G, Khoo L, et al. Can HRCT be used as a marker of airway remodelling in children with difficult asthma? Respir Res 2006; 7: 46.

10 Martínez TM, Llapur CJ, Williams TH, et al. Highresolution computed tomography imaging of airway disease in infants with cystic fibrosis. Am J Respir Crit Care Med 2005; 172: 1133-1138.
11 Klein J, Chitayat D, Koren G. Hair analysis as a marker for fetal exposure to maternal smoking. N Engl J Med 1993; 328: 66-67.

12 Nakano Y, Wong JC, de Jong PA, et al. The prediction of small airway dimensions using computed tomography. Am J Respir Crit Care Med 2005; 171: 142-146.

13 de Jong PA, Nakano Y, Hop WC, et al. Changes in airway dimensions on computed tomography scans of children with cystic fibrosis. Am J Respir Crit Care Med 2005; 172: 218-224.

14 Hedlund LW, Vock P, Effmann EL. Evaluating lung density by computed tomography. Semin Respir Med 1983; 5: 76-87.

15 Jones M, Castile R, Davis S, et al. Forced expiratory flows and volumes in infants. Normative data and lung growth. Am J Respir Crit Care Med 2000; 161: 353-359.

16 de Jong PA, Nakano Y, Lequin MH, Tiddens HA. Dose reduction for $\mathrm{CT}$ in children with cystic fibrosis: is it feasible to reduce the number of images per scan? Pediatr Radiol 2006; 36: 50-53.

17 de Jong PA, Ottink MD, Robben SG, et al. Pulmonary disease assessment in cystic fibrosis: comparison of CT scoring systems and value of bronchial and arterial dimension measurements. Radiology 2004; 231: 434-439.

18 Saglani S, Malmström K, Pelkonen AS, et al. Airway remodeling and inflammation in symptomatic infants with reversible airflow obstruction. Am J Respir Crit Care Med 2005; 171: 722-727.

19 Saglani S, Payne DN, Zhu J, et al. Early detection of airway wall remodeling and eosinophilic inflammation in preschool wheezers. Am J Respir Crit Care Med 2007; 176: 858-864.

20 Elliot J, Vullermin P, Robinson P. Maternal cigarette smoking is associated with increased inner airway wall thickness in children who die from sudden infant death syndrome. Am J Respir Crit Care Med 1998; 158: 802-806.

21 Hanrahan JP, Tager IB, Segal MR, et al. The effect of maternal smoking during pregnancy on early infant lung function. Am Rev Respir Dis 1992; 145: 1129-1135.

22 Hoo AF, Henschen M, Dezateux C, Costeloe K, Stocks J. Respiratory function among preterm infants whose mothers smoked during pregnancy. Am J Respir Crit Care Med 1998; 158: 700-705.

23 Frey U, Jackson AC, Silverman M. Differences in airway wall compliance as a possible mechanism for wheezing disorders in infants. Eur Respir J 1998; 12: 136-142.

24 Sekhon HS, Jia Y, Raab R, et al. Prenatal nicotine increases pulmonary $\alpha 7$ nicotinic receptor expression and alters fetal lung development in monkeys. J Clin Invest 1999; 103: 637-647.

25 Coxson HO, Mayo JR, Behzad H, et al. Measurement of lung expansion with computed tomography and comparison with quantitative histology. J Appl Physiol 1995; 79: 1525-1530.

26 Tepper RS, Williams T, Kisling J, Castile R. Static compliance of the respiratory system in healthy infants. Am J Respir Crit Care Med 2001; 163: 91-94. 